\title{
Damping Ratio Analysis of a Silicon Capacitive Micromechanical Accelerometer
}

\author{
Yuming Mo*, Lianming Du, Bingbing Qu, Bo Peng, Jie Yang \\ Institute of Electronic Engineering, China Academy of Engineering Physics, Mianyang, China \\ Email:*moym08@163.com
}

How to cite this paper: Mo, Y.M., Du, L.M., Qu, B.B., Peng, B. and Yang, J. (2017) Damping Ratio Analysis of a Silicon Capacitive Micromechanical Accelerometer. Wireless Sensor Network, 9, 178-188. https://doi.org/10.4236/wsn.2017.95010

Received: March 30, 2017

Accepted: May 24, 2017

Published: May 27, 2017

Copyright ( 2017 by authors and Scientific Research Publishing Inc. This work is licensed under the Creative Commons Attribution International License (CC BY 4.0).

http://creativecommons.org/licenses/by/4.0/

\section{(c) (i) Open Access}

\begin{abstract}
In most MEMS devices, the moving micro-structures are surrounded by air which significantly affects their dynamics behaviors. The correct prediction of the squeeze-film air damping ratio is essential in MEMS (Micro-Electro-Mechanical System) devices design. In the paper, a static test is proposed to measure the squeeze-film air damping ratios of capacitive MEMS accelerometer under different pressures. The unsealed chip of capacitive accelerometer is placed in vacuum extraction equipment and an open loop circuit is developed to apply step signal in the test. By charging the pressure and measuring the overshoot $M_{p}$ and the settling time $t_{s}$ from the time response of the system, the damping ratio $\xi$ under different pressures can be calculated. Finite element method (FEM) based on the modified Reynolds equation is utilized to simulate the transient response of the micro-structure. Good correlation between experiment and FEM analysis is obtained. The proposed static test in this paper provides a new method to more easily measure the dynamic performances of micro-structures under various pressures.
\end{abstract}

\section{Keywords}

MEMS, Accelerometer, Damping Ratio, Experiment, Finite Element Analysis

\section{Introduction}

With the development of micro fabrication, Micro-Electro-Mechanical-System (MEMS) devices such as MEMS sensors, MEMS actuators and micro optical devices are widely used in aviation, aerospace, biomedicine and so on. The most notable characteristic of MEMS devices is that their structures are a few microns in size and separated by micron-sized gaps which are filled with fluid (mostly is air). As to micro-structure, the decrease in size leads to high surface-mass ratios and high surface forces that can mostly be neglected in macro-structures. Therefore, the dynamic behaviors of micro-parts in MEMS devices are tremen- 
dously affected by the fluid damping. The dominant damping in MEMS devices is the squeezed film air damping causing by air squeezed in and out of the gaps between the moving parts and the stationary parts. As the surface-mass ratios increase, viscous damping of the ambient fluid increases rapidly leading to significant energy dissipation. Namely, the performance or the quality factor of MEMS devices is largely determined by the squeezed film air damping. As a result, the design of the squeezed film damping becomes a crucial problem in MEMS.

Newell [1] firstly observed that air damping increases rapidly as the surface-mass ratio increases when a cantilever resonator is miniaturized and presented the formulas of $\mathrm{Q}$ under three atmospheric pressure regions. Soon afterwards, much attention is paid to the investigation of squeezed film air damping in MEMS [2] [3] [4] [5]. The squeeze film damping in MEMS devices varies with different pressures. At low pressure, the models for the flow of rarefied air in a narrow gap $(10 \mu \mathrm{m})$ have been presented in literatures [6] [7] [8] [9]. To avoid air damping, using a vacuum packaging to enable MEMS devices working in low-pressure environment is a feasible way. The other way is to optimize the moving parts, such as perforating the plate. Zelong Zhou et al. [10] used the lava pipe model to obtain the velocity of air flow to modify the conventional Reynolds equation to investigate the dynamic behaviors of thick perforated plate. By using theoretical models and experiments, Eung-Sam Kim et al. [11] paid their attention to investigate how the size and number of perforation influence the squeeze film damping. Vacuum packaging and perforated plate have been proved to be useful method to reduce squeeze film damping in MEMS devices.

When a MEMS chip is packaged under a certain pressure, the dynamic response of it is generally aimed at defining its behavior in the working conditions and its performance in terms of the undamped natural frequency, quality factor (Q). The $\mathrm{Q}$ of a MEMS device is the most important properties which provide immediate quantification of energy dissipations and it is largely determined by the squeeze film damping. Hence, how to easily and accurately examine the squeeze film damping becomes essential.

In this paper, a static test based on an open loop circuit is presented to measure the squeeze film damping ratio of a capacitive MEMS accelerometer with a 2 $\mu \mathrm{m}$ air gap under different air pressures. FEM, used to solve the modified Reynolds equation, is applied to simulate the transient response. There is good agreement between experimental result and finite element analysis.

\section{Theoretical Background of the Analysis}

\subsection{Underdamped System}

Based on a bulk micromachining technology, the capacitive MEMS accelerometer that consists of three silicon waters is illustrated in Figure 1. By monitoring the displacement of the proof mass attached to the spring, the MEMS accelerometer can sense the applied acceleration. Then the governing equation of this mass-spring system is given by 


$$
M \ddot{x}+C \dot{x}+K x=M a
$$

where $x$ is the displacement of the mass, $M$ is the proof mass, $C$ is the damping coefficient and $K$ is the stiffness of spring.

By applying Laplace transform is Equation (1), the transfer function of the MEMS accelerometer is obtained

$$
H(s)=\frac{\omega_{n}^{2}}{s^{2}+2 \xi \omega_{n} s+\omega_{n}^{2}}
$$

where $\xi=D / \sqrt{K M}$ is damping ratio and $\omega_{n}=\sqrt{K / M}$ is the undamped natural frequency. When designing the control circuit and parameters of the structure of the MEMS chip, the undamped natural frequency and the damping ratio needs to be confirmed by test.

The motion of the mass-spring-damping system in MEMS accelerometer is equivalent to a second-order system. For a second-order underdamped system $(\xi<1)$, its requirements for a step response are expressed in terms of some standard quantities illustrated in Figure 2. The time history of the step response of a second-order system found from the inverse Laplace transform of $H(s) / s$ is

$$
x(t)=1-\frac{1}{\sqrt{1-\xi^{2}}} \mathrm{e}^{-\xi \omega_{n} t} \sin \left(\omega_{d} t+\beta\right)
$$

Then, the overshoot $M_{p}$ which is defined as the maximum amount the system overshoots its final value divided by its final value can be calculated when the derivative is zero. From calculus we have this formula

$$
M_{p}=\mathrm{e}^{\frac{-\pi \xi}{\sqrt{1-\xi^{2}}}}
$$

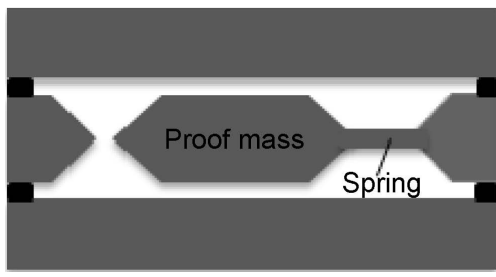

Figure 1. Capacitive MEMS accelerometer cross section.

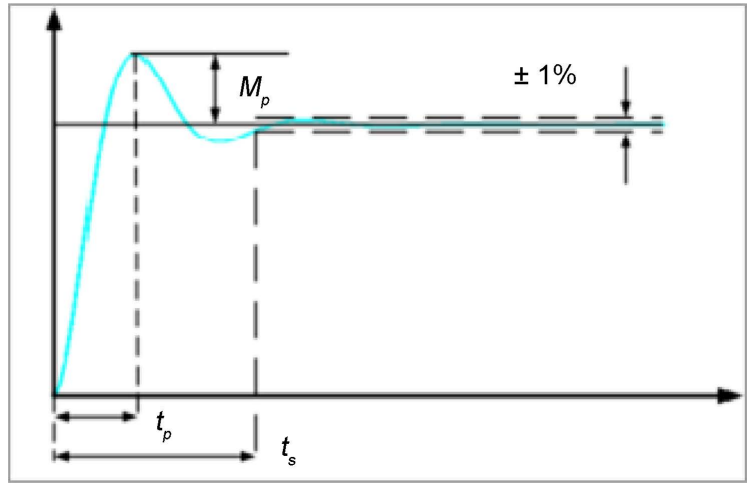

Figure 2. Definition of settling time $t_{s}$ and overshoot $M_{p}$. 
From Equation (4), the damping ratio can be calculated by taking the logarithm on both sides

$$
\xi=\frac{\left|\ln \left(M_{p}\right)\right|}{\sqrt{\pi^{2}+\left[\ln \left(M_{p}\right)\right]^{2}}}
$$

As the damping ratio increases, the overshoot does not exist. Therefore, the damping ratio can still be calculated from its settling time. For a second-order underdamping linear system, the envelope curve of its step response is

$$
x(t)=1 \pm \frac{\mathrm{e}^{-\xi \omega_{n} t}}{\sqrt{1-\xi^{2}}}
$$

The template is used to format your paper and style the text. All margins, column widths, line spaces, and text fonts are prescribed; please do not alter them. You may note peculiarities. For example, the head margin in this template measures proportionately more than is customary. This measurement and others are deliberate, using specifications that anticipate your paper as one part of the entire journals, and not as an independent document. Please do not revise any of the current designations.

The settling time $\left(t_{s}\right)$ is that the system takes to settle down to steady-state and it is define as $|x(t)-x(\infty)| \leq \Delta \cdot x(\infty), \Delta=0.02$ or $\Delta=0.05$.

- When $\Delta=0.02, \xi \approx \frac{4.2}{t_{s} \omega_{n}}$

- When $\Delta=0.05, \xi \approx \frac{3.5}{t_{s} \omega_{n}}$

Therefore, by measuring $M_{p}$ and $t_{s}$ of the step response of the mass-springdamping system, the damping ratios caused by squeeze film air under different pressures are obtained. Furthermore, the quality factor of the MEMS accelerometer can be approximatively calculated by $Q=1 / 2 \xi$.

\subsection{Overdamped System}

When $\xi>1$, the characteristic equation of second-order system is

$$
s^{2}+2 \xi \omega_{n} s+\omega_{n}^{2}=\left(s+\frac{1}{T_{1}}\right)\left(s+\frac{1}{T_{2}}\right)=0
$$

where

$$
T_{1}=\frac{1}{\omega_{n}\left(\xi-\sqrt{\xi^{2}-1}\right)}, T_{2}=\frac{1}{\omega_{n}\left(\xi+\sqrt{\xi^{2}-1}\right)}
$$

The overdamped step response of second-order system is shown in Figure 3. Let $T_{1} / T_{2}=n$ and use $5 \%$ as a reasonable measure. We will have

- When $T_{1} / T_{2}=1$, then $t_{s}=4.75 T_{1}$ and $\xi=1$;

- When $T_{1} / T_{2}=4$, then $t_{s} \approx 3.3 T_{1}$ and $\xi \approx 1.25$;

- When $T_{1} / T_{2}>4$, then $t_{s} \approx 3 T_{1}$ and $\xi>1.25$;

Let $t_{s}=n T_{1}$, from Equation (8), the damping ratio will be obtained from cal- 
culus

$$
\xi=\frac{1}{2}\left(\frac{t_{s} \omega_{n}}{n}+\frac{n}{t_{s} \omega_{n}}\right)
$$

The settling time can be measured from the step response of the accelerometer and the squeeze film air damping ratio is obtained using Equation (9).

\section{Test Setup and Results}

\subsection{Test Setup}

In order to measure the step response of the MEMS accelerometer, an open loop circuit is designed shown in Figure 4. The test system consists of a bias voltages circuit, excitation signal source, signal generator, $\mathrm{C}-\mathrm{V}$ converter, oscilloscope and a vacuum chamber.

When testing, the voltage signal from the signal generator is applied to the top electrode. The vacuum test bench is used to change the ambient pressure in the capacitive MEMS accelerometer. By pulling the proof mass to its equilibrium position and releasing under different air pressures, the step response of the MEMS accelerometer is tested. From the plots of the transient response of the system recorded by the oscilloscope, the overshoot $M_{p}$ and the settling time $t_{s}$ can be measured. And then, the squeeze film air damping ratio can be calculated. In this way, the electrostatic stiffness is also efficiently avoided. Most notably, due to the application of phase sensitive detection, the excitation signal frequency must be larger enough than the frequency of signal generator. In the test the excitation signal frequency is $100 \mathrm{kHz}$.

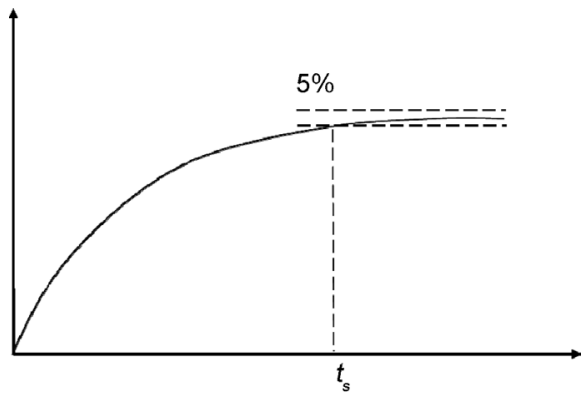

Figure 3. Response of overdamped system.

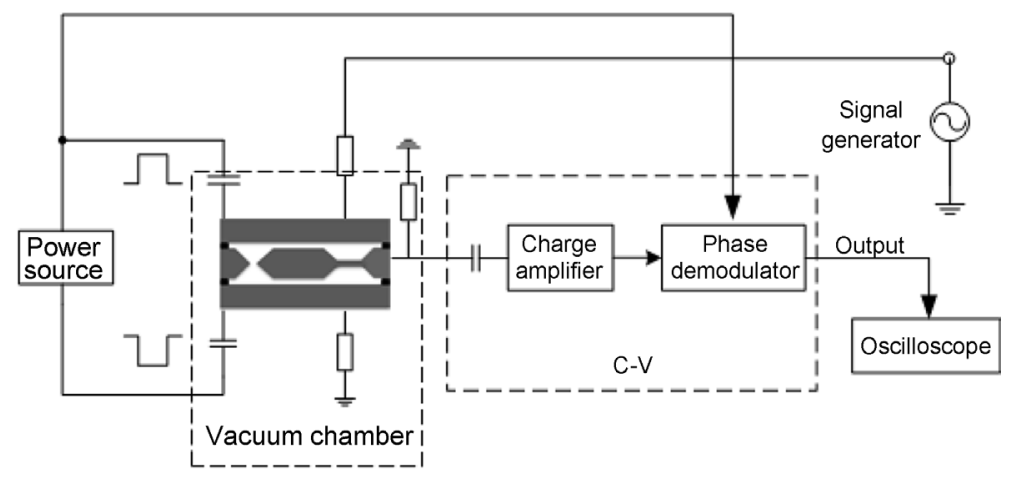

Figure 4. Test setup. 


\subsection{Test Results}

By changing the ambient pressure in capacitive MEMS accelerometer using the vacuum chamber, the squeeze film air damping ratio at $100 \mathrm{~Pa}, 300 \mathrm{~Pa}$, $1600 \mathrm{~Pa}, 3000 \mathrm{~Pa}$ and room pressure. The plots of transient response of the capacitive MEMS accelerometer are shown is Figure 5. The overshoots $M_{p}$, settling time $t_{s}$ and the squeeze film are damping ratio $\xi$ calculated from the measured overshoot are listed in Table 1.

\section{Finite Element Analysis}

\subsection{Govern Equation}

The two basic damping mechanisms in inertial MEMS are slide and squeeze damping. Slide damping happens when a suspended plate is moving parallel to the substrate and it is not considered in this paper. Squeeze damping occurs when a parallel plate is moving vertical to the substrate. The motion increases the pressure and squeezes the gas out of the gap or decreases the pressure and draws the gas into the gap. The situation of capacitive MEMS accelerometer considered in this paper is demonstrated in Figure 6.

For macro-structures, the viscous and inertial effect both affects the behavior of squeeze film. However, for micro-structure in MEMS devices, inertial effect

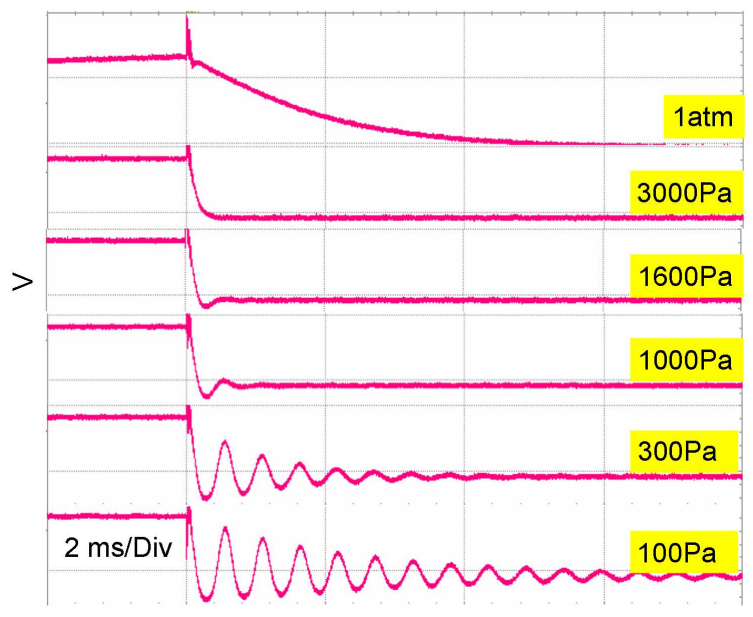

Figure 5. Plots of the step response of the capacitive MEMS accelerometer at different pressures.

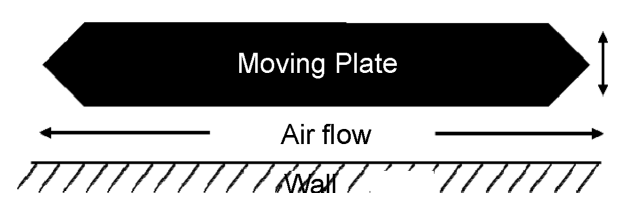

Figure 6. Squeeze film air damping.

Table 1. Test results of under different pressures $(\mathrm{Pa})$.

\begin{tabular}{ccccccc}
\hline Pressure & 100 & 300 & 1000 & 1600 & 3000 & 1 atm \\
\hline$\xi$ & 0.172 & 0.189 & 0.344 & 0.457 & 1.003 & 16.038 \\
\hline
\end{tabular}


can be negligible. Therefore, the squeeze film air damping problems encountered in the capacitive MEMS accelerometer is govern by the Reynolds equation which has the following formula [12]

$$
\frac{\partial}{\partial x}\left(\rho \frac{h^{3}}{\mu} \frac{\partial P}{\partial x}\right)+\frac{\partial}{\partial y}\left(\rho \frac{h^{3}}{\mu} \frac{\partial P}{\partial y}\right)=12 \frac{\partial(h \rho)}{\partial t}
$$

Due to the small dimensions in MEMS devices, the temperature variation is negligible. With the isothermal condition, air density $\rho$ is proportional to its pressure $P$. Therefore, we have the nonlinear Reynolds equation commonly used in MEMS for isothermal squeeze film damping of compressible gas

$$
\frac{\partial}{\partial x}\left(\frac{P h^{3}}{\mu} \frac{\partial P}{\partial x}\right)+\frac{\partial}{\partial y}\left(\frac{P h^{3}}{\mu} \frac{\partial P}{\partial y}\right)=12 \frac{\partial(h P)}{\partial t}
$$

where pressure $P$ consists of two components: $P=P_{a}+p ; P_{a}$ is the ambient pressure and $P$ is the deviatory pressure caused by the squeeze air film effect; $h$ is the height of the air gap and $\mu$ is the dynamic viscosity.

When a plate is moving perpendicular to the substrate, the air gap height $h$ and dynamic viscosity $\mu$ are not functions of position. Equation (11) is simplified as

$$
\frac{\partial}{\partial x}\left(P \frac{\partial P}{\partial x}\right)+\frac{\partial}{\partial y}\left(P \frac{\partial P}{\partial y}\right)=\frac{12 \mu}{h^{3}} \frac{\partial(h P)}{\partial t}
$$

When the ambient pressure $P_{a}$ is small, the rarefaction effects need to be taken into account. A few approaches are reported to model the rarefaction effects for squeezed $\mathrm{lm}$ damping problems under a wide range of ambient pressures [13]. To model such phenomena, Reynolds equation need to be modified [7] [8] [13], That is, by replacing the dynamic viscosity $\mu$ in Reynolds equation with the effective viscosity $\mu_{\text {eff }}$ The commonly used effective viscosity is given by Fukui [14] and Veijola et al. [15] which has the following formula

$$
\mu_{\text {eff }}=\frac{\mu}{1+9.638 K_{n}^{1.159}}
$$

where $K_{n}$ is the Knudsen number. In this paper, the formula of effective viscosity is defined as Equation (14):

$$
\mu_{\text {eff }}=\frac{\mu}{1+9.638 \alpha K_{n}^{1.159}}
$$

where $\alpha$ is the surface correcting coefficient [8] and $\alpha=0.6$ in this paper. For fluid in MEMS devices, the Knudsen number decides the fluid flow model of the system and is estimated by

$$
K_{n}=\frac{\lambda}{h_{0}}
$$

there, $\lambda$ is the gas-specific mean free and can be estimated by $\lambda=P_{0} \lambda_{0} / P_{a}$, $\lambda_{0}$ is the mean free path under pressure $P_{0} ; h_{0}$ is the gap height. For very narrow gap and low pressure, the Knudsen number increases and the fluid flow transits from continuum flow to rarefied flow. 


\subsection{Finite Element Model}

In this paper, the transient response of the capacitive MEMS accelerometer was computed by using COMSOL Multiphysics v5.1 software. Figure 7 shows the model of thin-film flow and some terms used in COMSOL Multiphysics v5.1.

For non-slip conditions at the proof mass and the bases, the modified Reynolds equation takes the form for a transient model:

$$
\begin{gathered}
\frac{\partial}{\partial t}\left(p_{\text {tot }} h\right)+\nabla_{t} \cdot\left(p_{\text {tot }} h \boldsymbol{v}_{\text {ave }}\right)-p_{\text {tot }} \boldsymbol{v}_{\omega} \cdot \nabla_{t} h=0 \\
\boldsymbol{v}_{\text {ave }}=\frac{1}{2} \boldsymbol{v}_{p, t}-\frac{h^{2} \mu_{\text {eff }}}{12} \nabla_{t} p_{f}
\end{gathered}
$$

where $p_{\text {tot }}=p_{A}+p_{f}, p_{A}$ is the ambient pressure, $p_{f}$ is the pressure developed as a result of the flow. $h$ is the gap height. In the up gap, $h=h_{1}+\boldsymbol{n}_{z} \cdot \boldsymbol{u}_{p}$; In the down gap, $h=h_{1}-\boldsymbol{n}_{z} \cdot \boldsymbol{u}_{p}$. In COMSOL Multiphysics v5.1 software, $p_{a}, h$ and $\mu$ need to be defined.

The finite element model of the spring-mass system is shown in Figure 8. The parameters of the spring-mass system are listed below:

- The air gap height: $h=2 \mu \mathrm{m}$

- Length of spring: $L_{s}=1385 \mu \mathrm{m}$

- Width of spring: $W_{s}=1452 \mu \mathrm{m}$

- Thickness of spring: $h_{s}=38 \mu \mathrm{m}$

- Length of proof mass: $L_{m}=1997 \mu \mathrm{m}$

- Width of proof mass: $W_{m}=1680 \mu \mathrm{m}$

- Thickness of proof mass: $h_{m}=380 \mu \mathrm{m}$

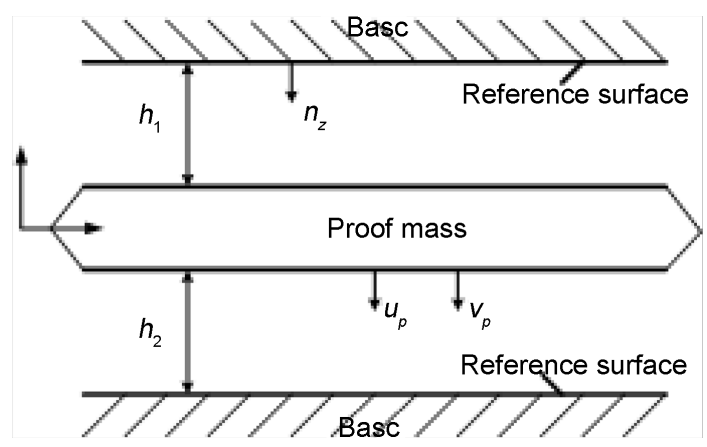

Figure 7. Typical configuration for thin-film flow in COMSOL.

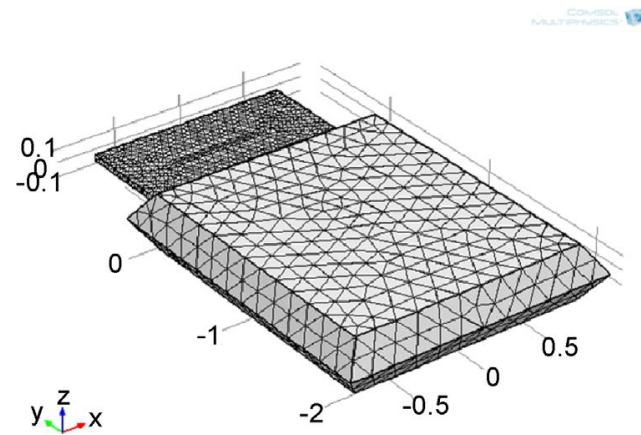

Figure 8. Finite element model of spring-mass system. 
Fixed constraint was applied on one side of the spring and the step load was applied on the proof mass. The thin film flow was defined on the up and down surface of the proof mass.

\subsection{Numerical Results}

The pressure distribution on the proof mass surfaces obtained from COMSOL Multiphysics is shown in Figure 9. As we can see in Figure 9, the maximum pressure is located in the forward part of the proof mass rather than the middle of it.

Figure 10 shows the dynamic response of the spring-mass system under

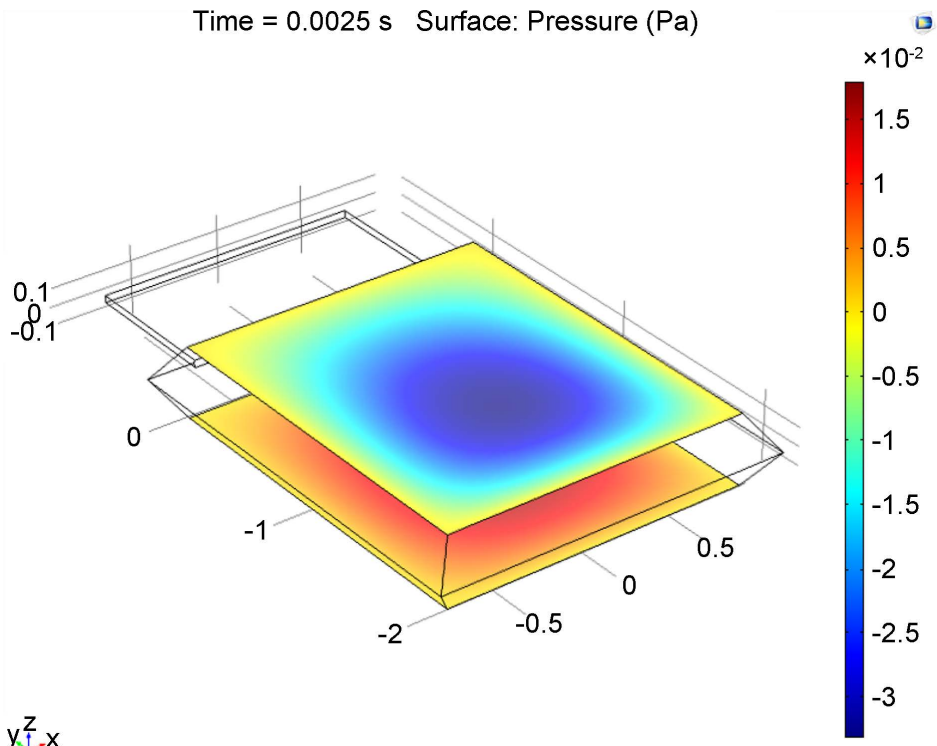

Figure 9. Pressure distribution on the surfaces of proof mass.

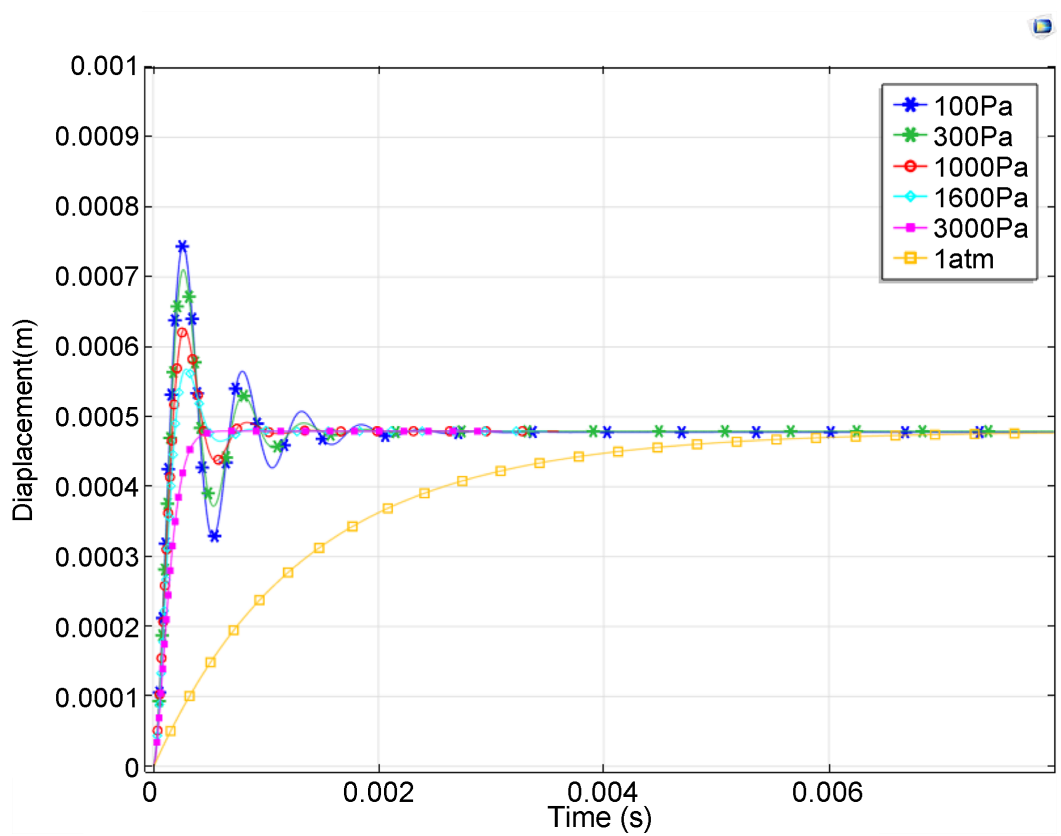

Figure 10. Dynamic response of the spring-mass system. 
Table 2. Numerical $\left(\xi_{c}\right)$ and test $\left(\xi_{t}\right)$ results of damping ratio.

\begin{tabular}{ccccccc}
\hline Pressure & 100 & 300 & 1000 & 1600 & 3000 & $1 \mathrm{~atm}$ \\
\hline$\xi_{t}$ & 0.172 & 0.189 & 0.344 & 0.457 & 1.003 & 16.038 \\
$\xi_{c}$ & 0.181 & 0.205 & 0.351 & 0.478 & 1.058 & 16.791 \\
\hline
\end{tabular}

standard earth gravity with different pressures. As the pressure increases, the oscillation times that the response requires to settle down to steady-state value decrease. When the pressure increases more than $3000 \mathrm{~Pa}$, the response curve does not oscillate any more. The curves show that the higher pressure leads to a larger damping ratio.

The damping ratios under different pressures obtained from test and computation are listed in Table 2. The comparison of numerical and test results show that the damping ratios of numerical result are all larger than those obtained from test. The reason is that the air cannot be squeezed out of the gap in the FEM model while some air is squeezed out of the gap in the test model. Therefore, the damping effect in the FEM model is more obvious than that in the test model. The largest error between the numerical and test results is $8.5 \%$ as it is acceptable.

\section{Conclusions}

The squeeze film air damping ratios in MEMS capacitive accelerometer is tested by using a special designed circuit and a vacuum chamber under different pressures in this paper. An assessment of dynamic response of the chip of a MEMS capacitive accelerometer is easily obtained by using this model.

The dynamic response of the chip of a MEMS capacitive accelerometer is analyzed by using finite element method. In the FEM model, the air viscosity is modified and numerical results are in good agreement with the test results within an acceptable error.

\section{References}

[1] Newell, W. (1968) Miniaturization of Tuning Forks. Science, 161, 1320-1326. https://doi.org/10.1126/science.161.3848.1320

[2] Chen, C.-S. and Kuo, W.-J. (2003) Squeeze and Viscous Dampings in Micro Electrostatic Comb Drives. Sensors and Actuators A, 107, 193-203.

[3] Mehner, I., Billep, D., et al. (1998) Simulation of Gas Damping in Microstructures with Nontrivial Geometries. Proceedings of the 11 th Annual International Workshop on Micro Mechanical Systems, Heidelberg, 25-29 January 1998, 172-177. https://doi.org/10.1109/memsys.1998.659749

[4] Hutcherson, S.M. (2004) Theoretical and Numerical Studies of the Air Damping of Micro-Resonators in the Non-Continuum Regime. Dissertation, G. W. Woodru School of Mechanical Engineering, Georgia Institute of Technology.

[5] Moeenfard, H. (2015) Analytical Modeling of Squeeze Film Damping in Dual Axis Torsion Microactuators. Surface Review and Letters, 22, 1550006-1-1550006-8. https://doi.org/10.1142/S0218625X15500067

[6] Brugdorfer, A. (1959) The Influence of the Molecular Mean Free Path on the Per- 
formance of Hydrodynamic Gas Lubricated Bearings. Journal of Basic Engineering, 81, 94-99.

[7] Hsia, Y.T. and Domoto, G.A. (1983) An Experimental Investigation of Molecular Rarefaction Effects in Gas Lubricated Bearings at Ultra Low Clearance. Journal of Lubrication Technology, 105, 130-130.

[8] Mitsuya, Y. (1993) Modified Reynolds Equation for Ultra-Thin Film Gas Lubrication Using 1.5-Order Slip-Flow Model and Considering Surface Accommodation Coefficient. Journal of Tribology, 115, 289-294. https://doi.org/10.1115/1.2921004

[9] Tan, Y., Lv, Y. and Pan, Z. (2012) The Analysis and Simulation of Squeeze Film Damping Characteristics of Low Air Pressure MEMS Accelerometer. IEE International Conference on Oxide Materials for Electronic Engineering, 351-354.

[10] Zhou, Z. and Wang, X. (2011) Analysis of Squeeze-Film Air Damping of Thick Perforated Plate in MEMS Device. Proceedings of the 6 th IEEE International Conference on NanalMicro Engineering and Molecular Systems, Kaohsiung, 20-23 February 2011, 131-133. https://doi.org/10.1109/nems.2011.6017312

[11] Kim, E.-S., Cho, Y.-H. and Kim, M.-U. (1999) Effect of Holes and Edges on the Squeeze Film Damping of Perforated Micromechanical Structures. 12 th IEEE International Conference on Micro Electro Mechanical Systems, Orlando, FL, 21 January 1999, 296-301.

[12] Darling, R., Hivick, C. and Xu, J. (1998) Compact Analytical Modeling of Squeeze Film Damping with Arbitrary Venting Conditions Using a Greens Function Approach. Sensors and Actuators A, 70, 32-41.

[13] Pantano, M.F. and Pagnotta, L. (2013) A Numerical Study of Squeeze-Film Damping in MEMS-Based Structures Including Rarefaction Effects. Frattura ed Integrità Strutturale, 23, 103-113.

[14] Fukui, S. and Kaneko, R. (1988) Analysis of Ultra-Thin Gas Film Lubrication Based on Linearized Boltzmann Equation: First Report-Derivation of a Generalized Lubrication Equation Including Thermal Creep Flow. Journal of Tribology, 110, 253 262. https://doi.org/10.1115/1.3261594

[15] Veijola, T., Kuisma, H., Lahdenpera, J. and Ryhanen, T. (1998) Equivalent-Circuit Model of the Squeezed Gas Film in a Silicon Accelerometer. Sensors and Actuators $A, 48,239-248$.

\section{Submit or recommend next manuscript to SCIRP and we will provide best} service for you:

Accepting pre-submission inquiries through Email, Facebook, LinkedIn, Twitter, etc. A wide selection of journals (inclusive of 9 subjects, more than 200 journals)

Providing 24-hour high-quality service

User-friendly online submission system

Fair and swift peer-review system

Efficient typesetting and proofreading procedure

Display of the result of downloads and visits, as well as the number of cited articles

Maximum dissemination of your research work

Submit your manuscript at: http://papersubmission.scirp.org/

Or contactwsn@scirp.org 